\title{
Network Psychological Status and Role Analysis Based on Theory of Personality Interaction
}

\author{
Tianlin Chen ${ }^{1}$, Lan Luo ${ }^{2, *}$ \\ ${ }^{1}$ Psychological Couseling Center, Jiangxi University of Traditional Chinese Medicine Nanchang, \\ 330004, China \\ ${ }^{2}$ School of Humanity, Jiangxi University of Traditional Chinese Medicine Nanchang, 330004, China
}

Keywords: Psychology measurement system; Interactive; Comprehensive fuzzy evaluation; Group measurement; Evaluation index

\begin{abstract}
With the increase of frequency users use network, the impact that network influenced on the psychological development is more and more big, especially the character interaction. In this paper, the principle and the theory of computer psychological measurement system has been used, the comprehensive analysis method of network psychological status and role analysis has been designed. The psychological interaction mathematical model has been designed using comprehensive fuzzy evaluation, and the QQ, Wechat, microblog, jingdong, taobao and online game users have been selected as interactive psychological measurement group, the group interactive mental health comprehensive evaluation has been made using computer psychological measurement system, and the group interaction mental health index has been got, which provides theoretical basis for information network research of psychology.
\end{abstract}

\section{Introduction}

Network, as a virtual space that free from the real world, gives people a drama stage. Internet users are free to choose the show's characters, props, and style through chat tools of QQ, Wechat, microblog, Momo etc, through network mall of taobao, jingdong, dangdang, through online games of martial arts, fantasy. Mathematical model of comprehensive fuzzy evaluation is adopted in this paper, system research on the network psychological and character interactive has been taken using computer psychological measurement system, and the network group users' psychology characteristics group has been got.

\section{The framework design of Personal interaction network psychology and character analysis}

The impact that Personality interaction effect on network psychology and role play is very large, and certain evaluation model and the computer measurement system are needed in this effect evaluation. We uses comprehensive fuzzy evaluation and computer measurement system for Internet users to evaluate the psychological interactivity in this paper, and we design the overall framework of the network psychological evaluation, as shown in figure 1.

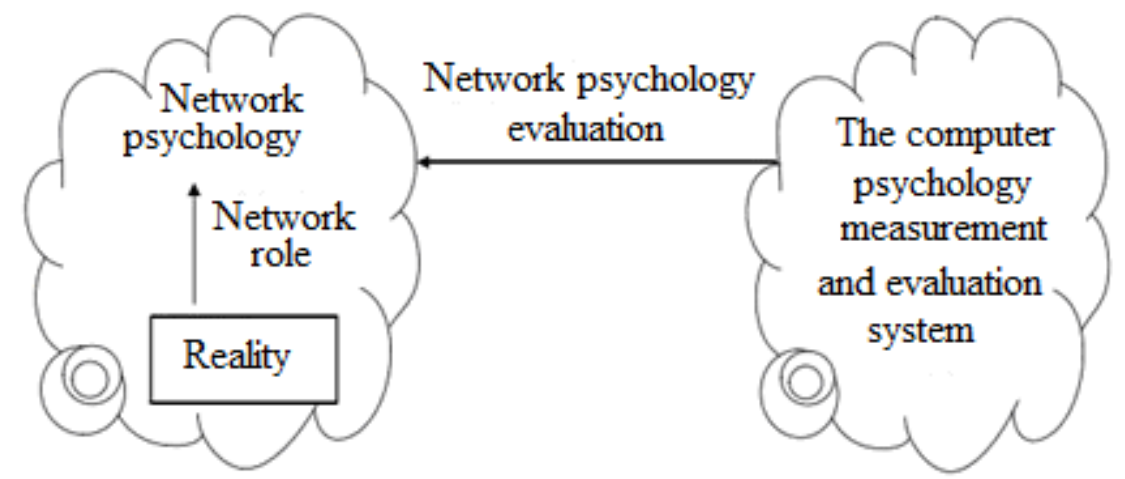

Fig.1 Internet users' network role interactive analysis frame diagram

Figure 1 shows the netizen network role psychology interactive analysis frame diagram, using the computer measuring system can realize comprehensive evaluation of group psychology, the 
selection of group rely on the psychological characteristics of real life, including the various kind of the role of network users, such as Wechat, microblog, QQ, jingdong, taobao and online users, etc. After selecting the network group, we uses the computer psychology measurement system to analyze psychological measurement data, then we can get the interactive analysis result of network group psychological role finally.

\section{The fuzzy control algorithm design of computer network psychology evaluation}

The basic domain of the fuzzy control algorithm of computer Network psychology evaluation is $\mathrm{Q}[-300,300]$, the basic domain of qc is [-200,200], the basic range of the resulting output V is [-300, 300]. First of all, we uses the algebraic expression to discrete fuzzy control algorithm of intelligent control system,

$$
y=\left[\frac{2 n}{j-i}\left(x-\frac{i+j}{2}\right)\right]
$$

In the formula (1), $x \in[i, j], \mathrm{n}$ is measuring group, Discrete degree of $\mathrm{Q}$ 、 qc and $\mathrm{V}$ is $\mathrm{n}=3$, we can get three discrete areas after discretization, $\mathrm{E}=\mathrm{EC}=\mathrm{U}=\{-3,-2,-1,0,1,2,3\}, 300$ sub-matrix relationship are obtained by discretization.

$$
R=R_{1} \cup R_{2} \cup \cdots \cup R_{i}(i=1,2, \cdots, 300)
$$

The output of the network psychology computer measurement results is shown in formula (3).

$$
U=(E \times E C) \bullet R
$$

We make a weighted average of the simulation output in the form of a weighted average, the fuzzy control of the final evaluation results is shown in formula (4),

$$
v=\frac{\sum_{i=0}^{300} v\left(z_{i j}\right) z_{j}}{\sum_{i=0}^{300} v\left(z_{i j}\right)}(i=j=1,2, \cdots, 300)
$$

In order to achieve the computer fuzzy measurement and evaluation of network group interaction psychology, we uses the Matlab fuzzy tool to design programming in this paper, the main Matlab algorithm is as follows,

$\mathrm{Q}=\left[\begin{array}{lll}0 & 0 ; 10 & 0\end{array}\right]$

$\mathrm{n}=2$;

$\mathrm{A}=[0-1 ; 10]$;

for $\mathrm{k}=1: 4$

$\mathrm{m}=5 * \mathrm{n}-4$;

$\mathrm{p}=\mathrm{Q}(1: \mathrm{n}-1, \mathrm{:})$;

$\mathrm{Q}(6: 5: \mathrm{m},:)=\mathrm{p}(2: \mathrm{n},:) ;$

$\mathrm{Q}(2: 5: \mathrm{m},:)=\mathrm{p}+\mathrm{d}$;

$\mathrm{Q}(3: 5: \mathrm{m},:)=\mathrm{p}+\mathrm{d}+0.7 * \mathrm{~d}^{*} \mathrm{~A}^{\prime}$;

$\mathrm{Q}(4: 5: \mathrm{m},:)=\mathrm{p}+2 * \mathrm{~d}+0.7 * \mathrm{~d} * \mathrm{~A}^{\prime}$;

$\mathrm{Q}(5: 5: \mathrm{m},:)=\mathrm{p}+2 * \mathrm{~d}$;

$\mathrm{n}=\mathrm{m}$;

end

\section{The computer measurement system design of Group interaction network psychology}

In order to verify the validity and reliability of the network psychological computer test fuzzy algorithm that designed in the second section of the paper, we uses the Matlab fuzzy toolbox to test network group interaction psychology samples in this paper, the toolkit interface is shown in Fig.2. 


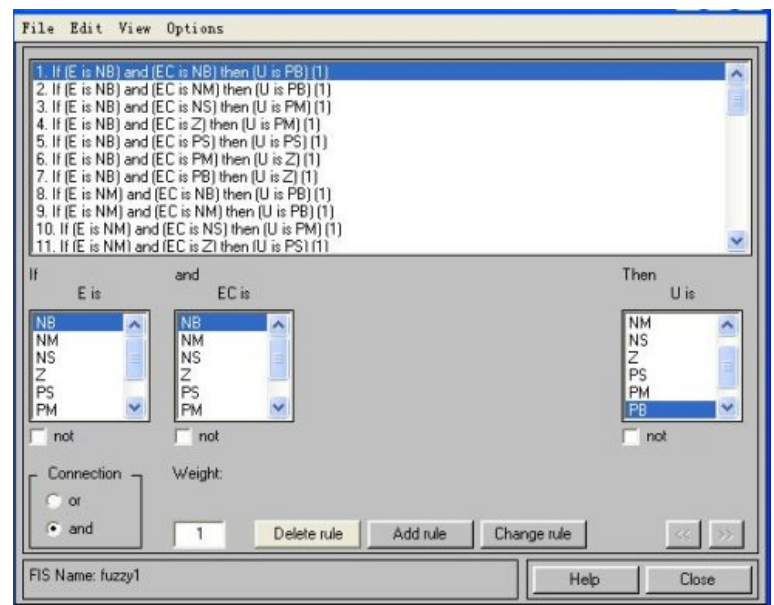

Fig. 2 The fuzzy control toolbox

Figure 2 shows the fuzzy control toolbox of Matlab software, Matlab toolbox can realize matrix operations, draw functions and data, realize algorithm, create users interface, connect other programming languages, etc., which is mainly used in engineering calculation, control design, signal processing and communication, image processing, signal detection, financial modeling design and analysis, etc.

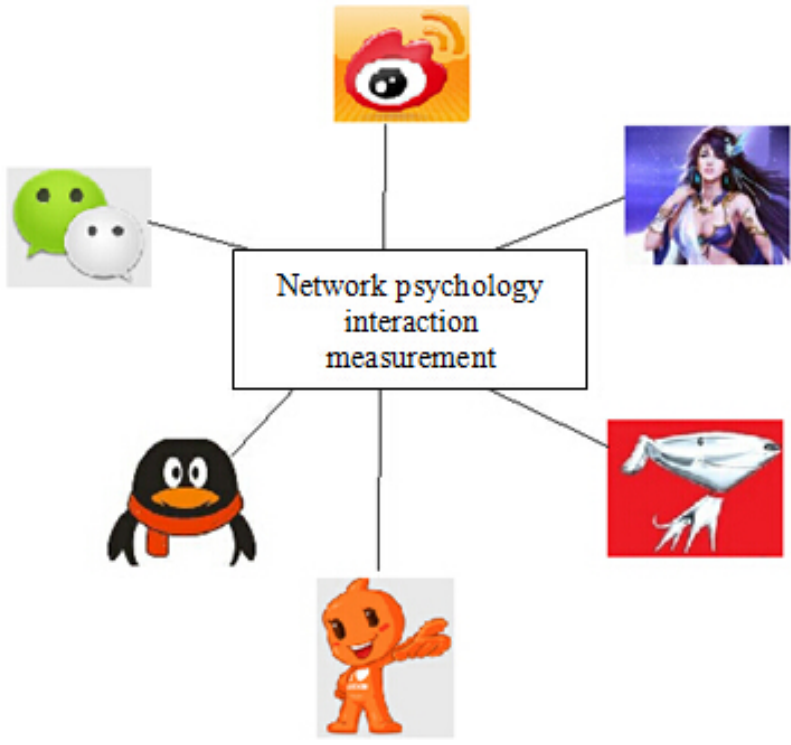

Fig. 3 network psychology measurement group selection Schematic diagram

As shown in figure 3, we have selected the 300 psychology measurement group, from Internet users such as Wechat, microblog, QQ, taobao, jingdong and online game users, we get comprehensive results of the psychological computer measurement through the comprehensive index psychological health test, the two-dimensional results are shown in figure 3.

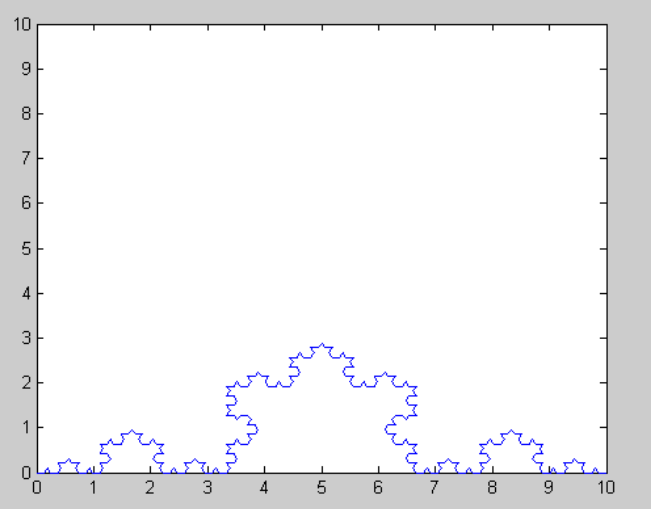

Fig.4 The two-dimensional evaluation of network group psychology role 
Figure 4 shows the two dimensional results schematic diagram of network group psychology role evaluation. It can be seen that two-dimensional plane result of group psychological measurement has obvious characteristics of fractal in the figure, and fractal pattern has obvious interaction, in order to further probe interactive as a result, we have drawn the figure of three dimensional result evaluation using Matlab.

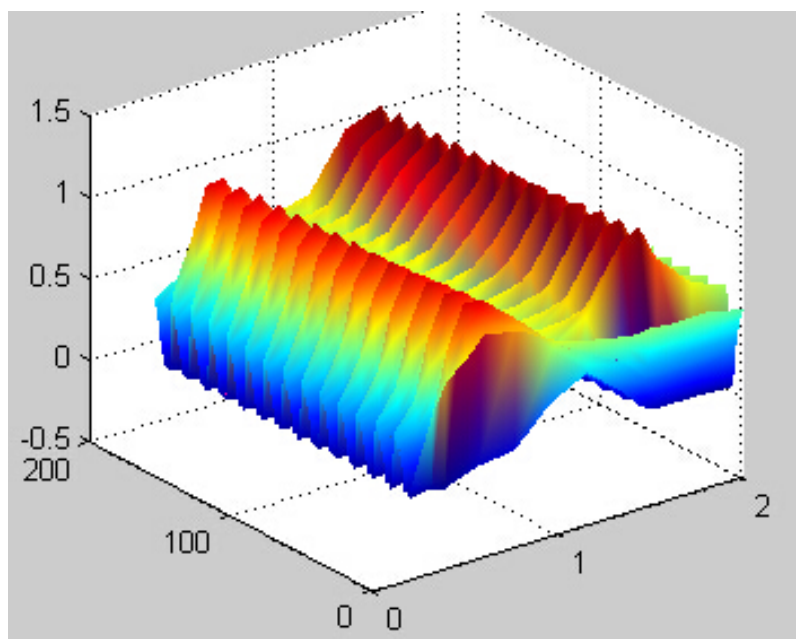

Fig. 5 The netizen psychology health comprehensive evaluation index

It shows that we use the computer psychology measurement system to take psychological test for two different age groups of Internet users in Fig.5, we eventually got health index as shown in figure 5 fitting curve, using fuzzy calculation toolbox, through the sample statistics of group psychological. By the graph, we can see that the psychological characteristics of netizens presents certain interactive distribution status under the action of the influence of group psychological interaction, health index presents two different peaks group, which have verified the interactive role of group psychology.

\section{Conclusion}

We have designed the network the basic domain and results domain of role psychology analysis using the integrated fuzzy intelligent control principle, and have used Matlab software to program control algorithm, and have design overall system using comprehensive fuzzy toolbox.

Then we have selected QQ, Wechat, microblog, jingdong, taobao and online game users as a interactive psychology measurement group, the network role psychology analysis of health comprehensive evaluation result is obtained by computing using the evaluation method presented in this paper, which has the vital significance community for network psychology research.

\section{References}

[1] Xie Yujin, Hu Shuxiang. The new attention of network man-machine contradictions and ideological and political education. Research on ideological education, No.6, (2010).

[2] Yuan Lin, Li Mei-qing. Rresearch on dilemma and crack of ideological and political education network transmission process management. Ideological and theoretical education Tribune, No.6, (2010).

[3] Jiang Xiaoli. Research on effectiveness evaluation system of college students' network ideological and political education. Journal of ideological education research, No.6, (2010).

[4] Zhi Fengying, Zhang Jinfu. The cultural perspective of Minority pressure study. Journal of psychological science. No.8, (2012)

[5] Yan Jiajia, Cui Yanting QuYan, Yang Xiaolei. Psychological elasticity theory research status at home and abroad . Journal of human resource management. No.6, (2012) 
[6] Miao Shenglong. Review of domestic research on psychological elastic orientation. Journal of social psychology science. No.5, (2012)

[7] Ji Huaimeng. Improved Apriori Algorithm Based on Frequency 2-item Set Support Matrix. Computer Engineering. Vol.39, No.11, (2013)

[8] Ma Ji. The scientific process of psychology testing technology. Journal of Chongqing college of liberal arts (social sciences edition), No.6, (2013)

[9] Sun Fengxiao, Ni Shihong, Xie Chuan. An improved Apriori algorithm based on matrix. Computer simulation. No.8, (2013)

[10] Wang Wei, Xin Zhiyong, Lei Li. The relationship between College students' values and their coping styles, mental health. Journal of renmin university of China education. No.04, (2012)

[11] Zhao Yandong, Zhou Chan. The analysis of the scientific research cooperation network of scientific research personnel, based on the research of individual center network perspective. Science research, No.7, (2011) 